\title{
Isogeneic comparison of primary and metastatic lung cancer identifies CX3CR1 as a molecular determinant of site-specific metastatic diffusion
}

\author{
FRANCESCO A. MAURI ${ }^{1,2 *}$, DAVID J. PINATO ${ }^{1,3 *}$, PRITESH TRIVEDI ${ }^{2}$, \\ ROHINI SHARMA ${ }^{1}$ and ROBERT J. SHINER ${ }^{4}$
}

${ }^{1}$ Division of Experimental Medicine and ${ }^{2}$ Department of Histopathology, Imperial College London, Hammersmith Campus,

Du Cane Road, London, UK; ${ }^{3}$ Department of Clinical and Experimental Medicine, Universitá degli Studi

del Piemonte Orientale ‘A. Avogadro', Via Solaroli, Novara, Italy; ${ }^{4}$ National Heart and Lung Institute,

Department of Respiratory Medicine, Hammersmith Hospital, Du Cane Road, London, UK

Received March 15, 2012; Accepted April 30, 2012

DOI: $10.3892 /$ or.2012.1818

\begin{abstract}
Metastatic diffusion is a major adverse prognostic determinant in lung cancer, that is ultimately responsible for significant morbidity, organ failure and death. Chemokine signaling pathways are known to guide site-specific metastatic spread in solid tumours. However, little is known about the contribution of CX3CR1 in the systemic dissemination of lung cancer. Syngeneic primary lung cancer/metastasis tissue microarray slides were constructed using 98 post-mortem specimens taken from patients with untreated lung cancer and immunostained for CX3CR1. Clinicopathological correlation between CX3CR1 expression and patient demographics, tumour histology, stage and pattern of metastatic spread was performed using $\chi^{2}$ test. CX3CR1 immunopositivity was significantly higher in non-small cell lung cancer (NSCLC) compared to small cell $(\mathrm{SCLC})$ primary $(\mathrm{p}<0.001)$ and secondary tumours ( $p<0.001$ ), with $>75 \%$ of the metastatic sites staining positively in NSCLC. CX3CR1 positivity was significantly associated with stage and number of metastatic sites $(\mathrm{p}=0.03)$. At patients' death CX3CR1-negative lung adenocarcinomas were more likely to have spread to the brain and the liver $(\mathrm{p}=0.01)$. CX3CR1 is upregulated in NSCLC metastatic disease and its expression in primary lung tumours relates inversely to organotropic spread of cancer cells to the brain and the liver.
\end{abstract}

Correspondence to: Dr Francesco A. Mauri, Department of Histopathology, Imperial College London, Hammersmith Campus, Du Cane Road, London W12 0HS, UK

E-mail: f.mauri@imperial.ac.uk

${ }^{*}$ Contributed equally

Key words: lung cancer, metastasis, chemokine, CX3CR1, fractalkine, CX3CL1

\section{Introduction}

Lung cancer is the leading cause of malignancy-related mortality worldwide (1). Metastatic dissemination is amongst the major determinants of adverse prognosis, affecting $\leq 50 \%$ of the patients presenting with small cell (SCLC) and non-small cell (NSCLC) lung carcinomas (2).

Despite the advancements achieved in the systemic treatment of lung cancer, the 5-year survival probability in patients with stage IV disease remains $<5 \%$ (3). Metastatic spread to vital organs such as the brain or the liver confers significant morbidity in patients with advanced lung cancer, ultimately leading to a worsening of clinical symptoms, organ failure and death (4).

It has long been recognized that the process of metastatic diffusion to distant organs does not happen at random, but rather reflects a complex interplay between the neoplastic cell clone and the target tissue (5). The invasion of the bloodstream by cancer cells is not sufficient to produce a secondary growth, that rather depends on their ability to survive in the bloodstream, arrest and extravasate into the target organ and secure independent growth capacity (6).

Among the factors that make the metastatic diffusion of cancer cells a highly selective process, an increasingly relevant role has been recognized for the chemokine system, a complex network of $>50$ different soluble glycoproteins and $20 \mathrm{G}$-protein coupled trans-membrane receptors that has been involved in the promotion of neoangiogenesis (7) as well as in the acquisition of invasive properties by tumour cells (8).

It has been shown that the constitutive production of different subtypes of chemokines may represent the molecular correlate which influences the preferential spread of tumour cells in specific anatomical sites of the body. It has been suggested that the chemokine secretion signature of a given tissue combined with the selective expression of the matched receptors by tumour cell clones might explain the site-specific spread of metastatic foci observed in solid tumours (9).

This concept is scientifically valid in lung cancer where the interaction between chemokine receptor CXCR4 and its ligand CXCL12 has been discovered to promote the metastatic potential 
in NSCLC both in vitro and in vivo (10), whereas the overexpression of CCR7 in primary lung cancer specimens is a predictor of a significant lymphotropic behavior (11).

Recently, an increasing scientific interest has been devoted to the study of the chemokine receptor CX3CR1, a seven spanning transmembrane protein that uniquely interacts with CX3CL1 or Fractalkine (FKN). It has been shown that tumour cell clones expressing CX3CR1 are induced to adhere to neuronal cells through the activation of $\beta$-integrins and focal adhesion kinase in pancreatic cancer (12). Likewise, overexpression of CX3CR1 in the primary tumour confers a 10 -fold increased risk for the development of brain metastases in node-positive breast cancer patients (13).

As the role of CX3CR1 in the metastatic spread of bronchogenic carcinoma is poorly defined, we designed this study in order to evaluate whether the expression of CX3CR1 in primary lung cancers specimens predicted the pattern of metastatic spread. In addition we evaluated the expression of CX3CR1 in metastatic lung cancer deposits. For this purpose, we adopted a tissue microarray (TMA) analysis approach taking advantage of an isogeneic collection matched primary and metastatic archival lung cancers obtained from post-mortem (PM) examinations of previously untreated lung cancer patients.

\section{Materials and methods}

Patients. The reports of 12,580 PM examinations performed at the Hammersmith Hospital between January 1970 and December 2005 were reviewed (Ethics Reference: 06/Q0406/ 154). Lung cancer was identified in 499 cases of whom 213 patients had not received any antemortem therapy for their cancer and had also undergone a complete post-mortem examination. Clinicopathological variables such as gender, age at death, tumour staging together with the number and distribution of metastatic sites were recorded. The absence of any chemotherapy or radiotherapy treatment was confirmed by a review of PM reports and medical notes. Tumour staging followed the TNM criteria 7th edition (14). Hematoxylin and eosin (H\&E) slides from these cases were reviewed by a board certified pathologist with expertise in pulmonary pathology (FAM) in order to confirm histotype classification as well as metastatic distribution. The quality of the primary lung and metastatic PM tissue was preliminary evaluated on newly cut $\mathrm{H} \& \mathrm{E}$ sections. In order to evaluate the suitability of the tissue for immunohistochemical studies, well-preserved specimens were immunostained for pan-cytokeratin MNF116 (Dako, Cambridge, UK) at 1:200 concentration after $0.1 \%$ trypsin in phosphate buffered saline incubation for $10 \mathrm{~min}$. PM specimens showing dubious or unsatisfactory MNF116 staining were discarded. Based on these quality criteria, we included a total number of 98 cases of primary lung cancer with 134 matched metastatic deposits.

Tissue microarray and immunohistochemistry. A syngeneic primary tumour/metastasis TMA was prepared as previously described (15). Three 1-mm cores were obtained from the most representative areas of the primary tumours and matched secondary lesions and re-embedded in microarray blocks. Antigen retrieval was carried out using standard procedures:
Table I. Clinicopathological features of the patients included in the study.

\begin{tabular}{lc}
\hline Patient characteristic & $\mathrm{N}=98$ \\
\hline Age at death, median (range) & $70(43-93)$ \\
& $73 / 25$ \\
Gender, M/F & \\
Histopathological classification & $65(66)$ \\
NSCLC & $30(31)$ \\
Squamous cell carcinoma & $32(33)$ \\
Adenocarcinoma & $3(2)$ \\
Mixed histology & $29(30)$ \\
SCLC & $4(4)$ \\
Large cell neuroendocrine carcinomas & \\
TNM stage & \\
II-III & $21(21)$ \\
IVa & $3(3)$ \\
IVb & $74(76)$ \\
SCLC stage & \\
Limited disease & $4(14)$ \\
Extensive disease & $25(86)$ \\
\hline
\end{tabular}

briefly, the sections were de-paraffinized in xylene, rehydrated in graded alcohols and heated in a microwave oven at $900 \mathrm{~W}$ for $20 \mathrm{~min}$ in citrate buffer at $\mathrm{pH} 6.0$ (16). Before immunostaining, slides were cooled at room temperature and endogenous peroxidase activity was suppressed by incubation with a $3 \%$ solution of $\mathrm{H}_{2} \mathrm{O}_{2}$ for 5 min. The primary antibody anti-CX3CR1 (Abcam ab8021, Cambridge, UK) was incubated overnight at the concentration 1:350.

The TMA sections were incubated with the secondary antibody for 1 hour at room temperature and then processed using the Polymer-HRP kit (BioGenex, San Ramon, CA, USA) with development in diaminobenzidine and Mayer's hematoxylin counterstaining. A pancreatic ductal adenocarcinoma tissue sample was used as external positive control during each reaction to confirm its specificity. Omission of the primary antibody and pre-absorption of the primary with the immunizing peptide were used as negative control reactions. This resulted in absence of staining in all cases.

CX3CR1 expression was scored as positive if tumour cells showed clear cytoplasmic immunostain as described before (17). Positivity in a single TMA core was considered enough to classify the case as positive. Two observers (F.A.M. and R.J.S.) blinded to the clinical data scored all the cases and results were found to be consistent.

Statistical analysis. Pearson's $\chi^{2}$ or Fisher's exact tests were used to elucidate any significant associations between categorical variables as appropriate. Associations were considered statistically significant at $\mathrm{p}$-value of $\mathrm{p}<0.05$. Analysis was performed using SPSS software version 
Table II. The relationship between CX3CR1 expression in the primary tumour and clinicopathological features at post-mortem examination.

\begin{tabular}{lccc}
\hline & $\begin{array}{c}\text { CX3CR1- } \\
\text { positive }\end{array}$ & $\begin{array}{c}\text { CX3CR1- } \\
\text { negative }\end{array}$ & P-value \\
\hline Gender, M/F & $38 / 9$ & $35 / 16$ & $\mathrm{NS}$ \\
$\begin{array}{l}\text { Age } \\
<65 / \geq 65\end{array}$ & $18 / 33$ & $10 / 37$ & $\mathrm{NS}$ \\
$\begin{array}{l}\text { Stage } \\
<\mathrm{IVb} / \mathrm{IVb}\end{array}$ & $16 / 31$ & $8 / 43$ & $0.03^{\mathrm{a}}$ \\
$\begin{array}{l}\text { Metastatic spread } \\
<6 / \geq 6 \text { sites }\end{array}$ & $42 / 5$ & $37 / 14$ & $0.04^{\mathrm{a}}$ \\
\hline
\end{tabular}

${ }^{\mathrm{a}} \mathrm{P}<0.05$, statistically significant.

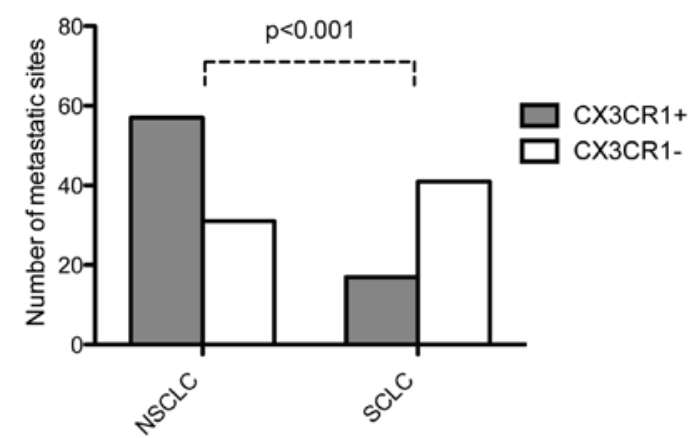

Figure 1. The proportion of CX3CR1 expression in NSCLC and SCLC metastatic deposits.

11.5 (SPSS Inc., Chicago, IL, USA) and GraphPad PRISM (GraphPad software Inc., La Jolla, CA, USA).

A

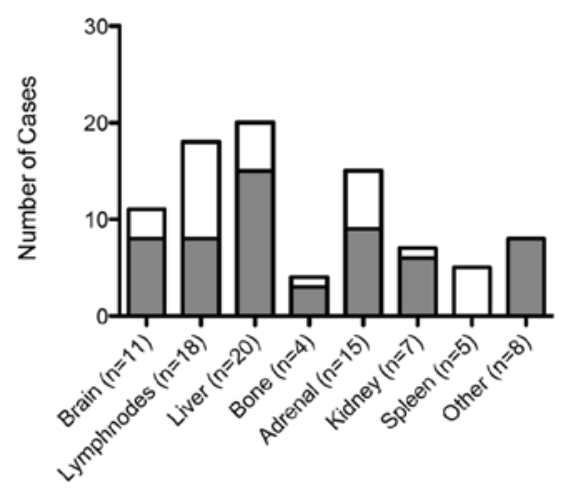

NSCLC

\section{Results}

Patients and tumour characteristics. The clinicopathological features reconstructed from the 98 PM included in the study are summarized in Table I. Briefly, the majority of patients were diagnosed with NSCLC (66\%). At the time of death, $76 \%$ of the subjects had evidence of distant metastases. SCLC cases displayed a more advanced spread to locoregional lymph nodes (26/29 cases, $90 \%$ versus $41 / 65,63 \%$; $\mathrm{p}<0.001)$ and to the liver $(21 / 29,72 \%$ versus $31 / 65 \mathrm{p}=0.02)$ when compared to NSCLC.

The expression of CX3CR1 in primary lung cancer and matched metastatic deposits. The frequency of CX3CR1 expression in the primary tumours was significantly higher in NSCLC (41/65, $63 \%)$ compared to SCLC $(2 / 29,7 \% \mathrm{p}<0.001)$. Histological subclassification revealed large cell neuroendocrine carcinoma $(4 / 4,100 \%)$ and squamous cell carcinomas $(27 / 30,93 \%)$ as being mostly CX3CR1 expressors when compared to adenocarcinomas $(16 / 32,50 \%, \mathrm{p}<0.001)$. The relationship between CX3CR1 immunopositivity in the primary tumour and the clinicopathological characteristics of our patient cohort is reported in Table II. The overall proportion of CX3CR1 immunopositive metastatic deposits was higher in NSCLC $(57 / 88,65 \%)$ compared to SCLC $(17 / 58,29 \%, \mathrm{p}<0.001)$ as shown in Fig. 1.

The distribution of CX3CR1 expression across the sampled metastatic sites is reported in Fig. 2. In NSCLC, atypical sites of metastatic spread including thyroid, heart, small bowel and skin were all positive to CX3CR1 $(8 / 8,100 \%)$, followed by $>75 \%$ of the kidney, liver, bone and brain deposits. All the sampled splenic metastases from NSCLC primaries were negative for CX3CR1 (5/5). In SCLC cases, 83\% (5/6) of the CNS metastatic sites were positive to CX3CR1 whereas only a minority of the other sites including locoregional lymph nodes $(2 / 14,14 \%)$ and liver $(4 / 14,28 \%)$ expressed this chemokine receptor. Adrenal gland metastases arising from SCLC were all negative for CX3CR1 (7/7).

The relationship between the expression of CX3CR1 in the primary tumour and the distribution of metastatic deposits is reported in Table III. Primary tumours lacking the expression of CX3CR1 were more likely to have spread to the brain $(\mathrm{p}=0.03)$ and liver $(\mathrm{p}=0.04)$ and histological subanalysis demonstrated this distribution to be typical of lung adenocar-

B

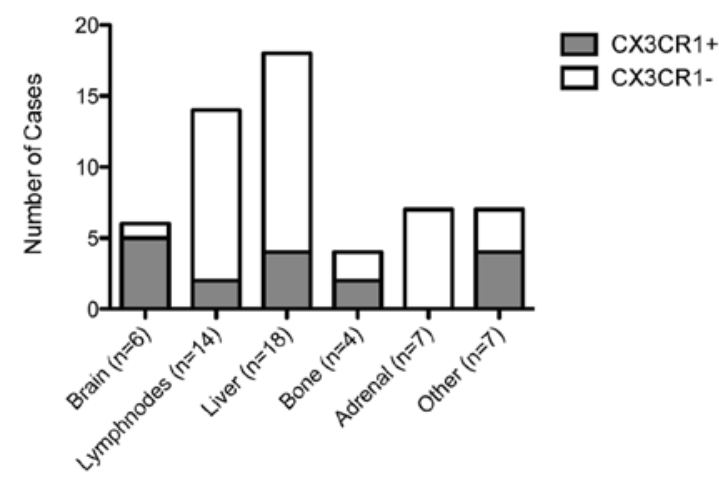

SCLC

Figure 2. Distribution of CX3CR1 expression in NSCLC and SCLC metastatic deposits across the sampled metastatic sites. 
Table III. The relationship between CX3CR1 expression across the different histotypes of primary lung cancer and the sitespecific metastatic spread.

\begin{tabular}{|c|c|c|c|c|c|c|c|c|c|c|c|c|}
\hline & & & & & & & & & & & & \\
\hline & & & & & & & & CR1 & & & & \\
\hline & + & - & P-value & + & - & P-value & + & - & $\mathrm{P}$-value & + & - & P-value \\
\hline Bra & & & & & & & & & & & & \\
\hline+ & 1 & 9 & $0.01^{\mathrm{a}}$ & 1 & 2 & NS & 1 & 5 & NS & 6 & 16 & $\mathbf{0 . 0 3}^{\mathrm{a}}$ \\
\hline - & 14 & 8 & & 22 & 5 & & 1 & 22 & & 41 & 35 & \\
\hline Liv & & & & & & & & & & & & \\
\hline+ & 4 & 13 & $0.01^{\mathrm{a}}$ & 5 & 2 & NS & 2 & 19 & NS & 21 & 34 & $0.04^{a}$ \\
\hline - & 11 & 4 & & 13 & 10 & & 0 & 8 & & 26 & 17 & \\
\hline Lyn & & & & & & & & & & & & \\
\hline+ & 6 & 9 & NS & 8 & 1 & NS & 2 & 21 & NS & 18 & 31 & $0.04^{\mathrm{a}}$ \\
\hline- & 9 & 8 & & 15 & 6 & & 0 & 6 & & 29 & 20 & \\
\hline Adr & & & & & & & & & & & & \\
\hline+ & 7 & 11 & NS & 3 & 2 & NS & 2 & 11 & NS & 17 & 24 & NS \\
\hline- & 6 & 8 & & 20 & 5 & & 0 & 16 & & 30 & 27 & \\
\hline Kid & & & & & & & & & & & & \\
\hline+ & 1 & 6 & NS & 3 & 1 & NS & 1 & 3 & NS & 6 & 10 & NS \\
\hline- & 14 & 11 & & 20 & 6 & & 1 & 24 & & 41 & 41 & \\
\hline Sple & & & & & & & & & & & & \\
\hline+ & 2 & 2 & NS & 3 & 0 & NS & 0 & 2 & NS & 5 & 4 & NS \\
\hline- & 13 & 15 & & 20 & 7 & & 2 & 25 & & 42 & 47 & \\
\hline Oth & & & & & & & & & & & & \\
\hline+ & 0 & 1 & NS & 2 & 0 & NS & 0 & 1 & NS & 3 & 2 & NS \\
\hline - & 15 & 16 & & 7 & 21 & & 2 & 26 & & 44 & 49 & \\
\hline
\end{tabular}

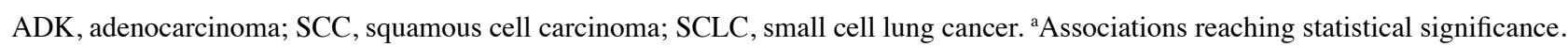

cinoma $(\mathrm{p}=0.01)$, but not of other histotypes. CX3CR1 status in the primary tumour correlated with N3 lymph node spread $(\mathrm{p}=0.04)$ and a significant association between CX3CR1 positivity within the primary tumour and in the secondary lymphatic spread was noted $(\mathrm{p}=0.03)$.

CX3CR1 positivity within the liver and adrenal deposits was associated with NSCLC histology $(p=0.001$ and $p=0.01$ respectively). Representative sections of CX3CR1-positive and -negative tumours are shown in Fig. 3.

\section{Discussion}

Previous research has shown that the differential expression of chemokines and their receptors can determine invasiveness and angiogenic potential of tumours and consequently is associated with a worse survival outcome in $\operatorname{NSCLC~}(7,18)$ as well as in SCLC $(19,20)$. In addition, the activation of specific chemokine signaling pathways may predict the organ orientation of the metastatic spread (21).

The expression of CX3CR1 has been previously reported in several tumours such as breast (13), prostate (22), pancreatic (12), ovarian cancer (23) and B-cell lymphoma (17). However, the precise role of this receptor in the malignant progression of lung cancer has not been addressed by previous research.

In our study we sought to determine whether CX3CR1 may represent a molecular factor involved in the metastatic spread of lung cancer. To test this hypothesis, we examined whether CX3CR1 expression in the primary tumour supported the metastatic site predilection of lung cancer cells on a series of untreated lung cancers. Interestingly, we found that primary tumours lacking CX3CR1 expression displayed a higher proportion of liver and brain metastatic deposits, and this was particularly significant in lung adenocarcinoma, a histotype with peculiar neurotropic and hepatotropic potential. 
CX3CR1-
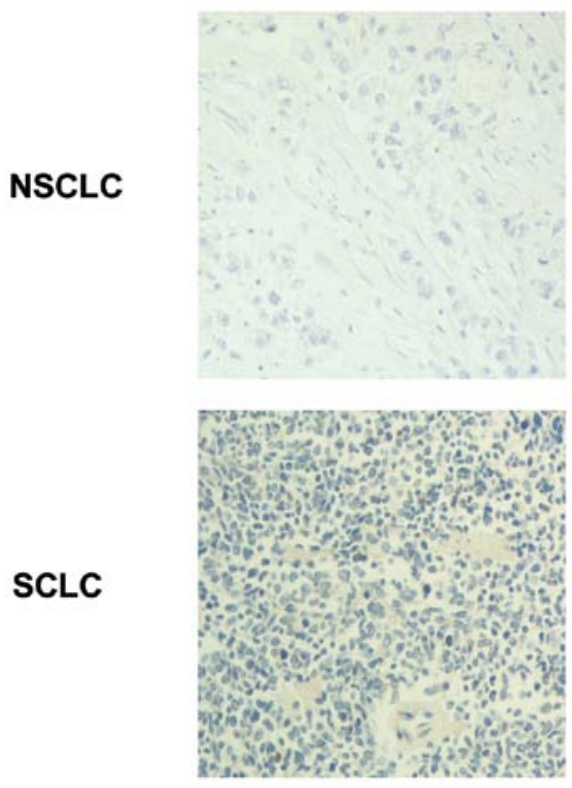

CX3CR1+
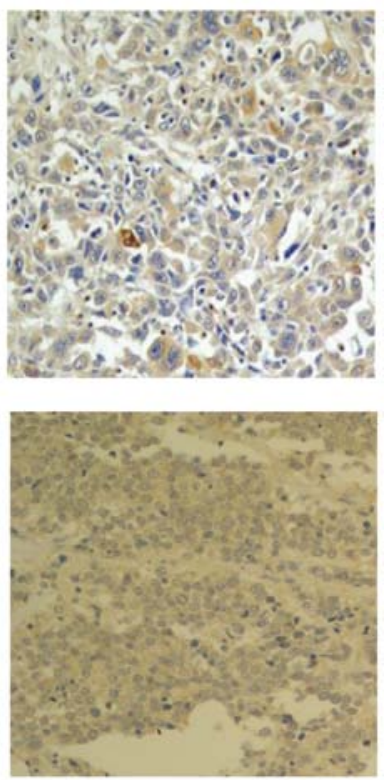

Figure 3. Expression of CX3CR1 by immunohistochemistry in representative sections of primary and metastatic lung cancer specimens grouped according to histology. Magnification x200.

Several published reports suggest that the aberrant activation of CX3CR1 is a molecular trait promoting the organotropic spread of primary tumours of the breast, pancreas and prostate to other vital organs including liver and brain.

However, in the specific context of lung cancer, we found that primary adenocarcinomas staining positively for CX3CR1 had a lesser tendency to diffuse to the liver and the brain. In keeping with the results described for hepatocellular carcinoma, where positivity to FKN and CX3CR1 reflects a less aggressive course of the disease (24), our findings let us infer that this molecular pathway can variably influence the process of metastatic spread across different tumour histotypes.

Since the molecular phenotype of lung primary tumours can differ significantly from that of their metastatic counterparts (25), we subsequently evaluated the distribution of CX3CR1 expression across the various sampled metastatic sites to further characterize its role in the systemic spread of the disease. Interestingly, the disproportion in CX3CR1 positivity we observed in the primaries was conserved in the matched metastatic deposits, in which NSCLC displayed higher expression rates over SCLC in all the sampled districts with the exception of the brain.

Strikingly, all the atypical sites of metastatic diffusion including myocardium, thyroid, small bowel and skin were positive to CX3CR1 in NSCLC, whereas SCLC adrenal secondaries did not express the receptor. As previous research in NSCLC has shown that CXC chemokines and their receptors are frequently subject to epigenetic silencing in primary tumours compared to normal bronchial epithelium (26), we may suppose that a reversal of such epigenetic control may be responsible for the significant degree of CX3CR1 positivity observed in the metastatic disease.

Furthermore, the high expression rates observed in specific districts of metastatic spread such as the liver and the central nervous system (CNS) support the hypothesis that the acquisi- tion of CX3CR1 positivity does not happen at random, but may rather represent a site-selective molecular event conferring growth advantage to the metastasized cell clone.

FKN is largely and constitutively expressed in the CNS (27) including in activated brain endothelial cells (28). It is hypothesized that FKN expression within the target tissue could provide a chemoattracting gradient promoting trans-endothelial migration of metastasizing tumour cells (29). According to this model, expression of CX3CR1 by epithelial tumours may favor the neurotropic dissemination of micrometastatic foci (30).

A similar mechanism is inherent in the pathogenesis of liver metastatic diffusion, a renowned poor prognostic trait in lung cancer (31), whereby the expression of FKN by hepatic sinusoidal cells may promote liver specific homing of pulmonary adenocarcinoma cells (24).

Since the activation of CX3CR1 is known to increase the phosphorylation of master regulators of cancer cell proliferation such as AKT and ERK1/2 (30), we speculate that the contribution of the FKN/CX3CR1 axis in the progression of lung cancer may be that it provides survival advantage signals to the metastasized tumour cells which secures independent growth potential within the brain parenchyma.

On the other hand, given the recognized role of FKN in the promotion of angiogenesis (32), it is reasonable to presume that the increased proportion of CX3CR1 positivity observed within the brain deposits may reflect a locally enhanced pro-angiogenic environment. It has been previously demonstrated that brain metastases do not share the same angiogenic phenotype of the corresponding primary NSCLC. In particular, the stronger production of vascular endothelial growth factor (VEGF) observed in the brain secondary tumours (25) may be mirrored by the consistent and potentially angiogenesis-driven up-regulation of CX3CR1 as reported in our study.

Irrespective of the mechanisms involved, the results of the present study suggest that CX3CR1 is involved in the clinical 
progression of NSCLC, being expressed in the majority of NSCLC metastatic deposits as well as in a predominant proportion of SCLC brain metastases.

Our data clearly show that the expression of FKN receptor, CX3CR1, is differentially distributed across the diverse histological subgroups of primary lung cancer, with almost two-thirds of the NSCLC being classified as receptor-positive. Moreover, CX3CR1 expression in the primary tumour correlates with advanced lymphatic spread and with the extent of metastatic dissemination independent of histology. Since oligometastatic patients have significantly better outcomes compared to patients with widespread metastatic diffusion (33), such an observation indirectly supports the role of CX3CR1 in driving the clinical behaviour of the disease, a concept that is further reinforced by the significant association found between receptor expression and tumour stage.

Although limited by the small sample size, our study is also the first to provide preliminary evidence that CX3CR1 expression may be involved in the pathophysiology of large cell neuroendocrine carcinomas an original finding that warrants further and more complete research in an adequately powered case series.

Since our study included advanced cases at the terminal stage of the disease we were unable to determine whether CX3CR1 positivity in the primary tumour can vary as a function of stage. On the other hand, our study design is peculiar in that it allowed us to find that up to three quarters of brain metastatic counterparts spread from both SCLC and NSCLC primaries expressed CX3CR1. Taken together, these findings suggest that this pathway might be a positive regulator in the progression of lung cancer.

We believe that the translational implications of this study are multifaceted. Firstly, routine pathological evaluation of CX3CR1 status in resected specimens of lung adenocarcinoma may represent a useful biomarker in the assessment of the metastatic potential of these tumours and allow for risk stratification of patients after curative treatment. Despite metastatic dissemination to the liver and the CNS is regarded as a common event in the natural history of bronchogenic carcinoma, there is a lack of predictors for the development of metastatic spread to these districts.

The CNS in particular represents the first and most common site of relapse after definitive treatment of non-small cell lung cancer (NSCLC) (34), eventually affecting $\leq 55 \%$ of the patients with locally advanced disease (35). Regardless of the histopathological classification, intracranial diffusion is invariably associated with reduced survival $(33,36)$.

Although a number of clinical predictors of metastatic spread to the CNS including nodal status and non-squamous histology (37) have been identified, the precise molecular mechanisms contributing to the neurotropic potential of NSCLC have not been fully elucidated.

Since CX3CR1-negative adenocarcinomas were more frequently associated with neurotropic spread, clinicians may incorporate this information to estimate the risk of intracranial progression and guide treatment decisions accordingly. Although such conclusion cannot be directly inferred from our retrospective data, prospective studies evaluating the impact of CX3CR1 on progression-free and overall survival are warranted.
A second, but not less important implication of our study relates to the role of the FKN/CX3CR1 axis as an emerging therapeutically meaningful pathway (38). Our study in fact provides preliminary evidence supporting a potential role for specific inhibitors of FKN/CX3CR1 in the setting of advanced lung carcinoma and possibly, as an adjuvant strategy to treat micrometastatic disease.

Clearly, the retrospective and single center nature of our study should be considered as a limitation when evaluating our results. Moreover, as our study was on post-mortem specimens, we could not assess the impact of CX3CR1 expression on patients' survival and staging at diagnosis. However, despite the small number of subjects included, the uniqueness of our case series has to be highlighted for two reasons. Firstly, all the enrolled patients did not receive systemic or radiation treatment which eliminates the possibility of treatment-induced changes in the expression of our marker. Secondly, collection of matched primary and multiple metastatic tissue is rarely achievable, in prospective studies.

In conclusion, we have shown that CX3CR1 is differentially expressed across NSCLC and SCLC, two histological subgroups of lung cancer with very different pathophysiology, clinical behavior and treatment strategies. We have indicated that CX3CR1 upregulation is a molecular trait shared by secondary brain tumours irrespective of histological categorization. Based on these results, CX3CR1 could not only represent a determinant in the biological aggressiveness of NSCLC but also a potential therapeutic target for advanced disease.

Moreover, negativity to CX3CR1 identifies a specific subgroup of primary tumours with a preferential tendency to metastasize to the brain and the liver, which suggests that CX3CR1 may be a potential biomarker of site-specific organotropic spread.

\section{Acknowledgements}

We would like to acknowledge John Brennan and Paul Nya for their help in the retrieval of tissue specimens. D.J.P. received support from Fondazione De Agostini for PhD studies.

\section{References}

1. Jemal A, Siegel R, Xu J and Ward E: Cancer statistics, 2010. CA Cancer J Clin 60: 277-300, 2010.

2. Cersosimo RJ: Lung cancer: a review. Am J Health Syst Pharm 59: 611-642, 2002

3. Fry WA, Phillips JL and Menck HR: Ten-year survey of lung cancer treatment and survival in hospitals in the United States: a national cancer data base report. Cancer 86: 1867-1876, 1999.

4. Sawaya R: Considerations in the diagnosis and management of brain metastases. Oncology (Williston Park) 15: 1144-1165, 2001.

5. Langley RR and Fidler IJ: Tumor cell-organ microenvironment interactions in the pathogenesis of cancer metastasis. Endocr Rev 28: 297-321, 2007.

6 . Fidler IJ: The pathogenesis of cancer metastasis: the 'seed and soil' hypothesis revisited. Nat Rev Cancer 3: 453-458, 2003.

7. Strieter RM, Belperio JA, Burdick MD, Sharma S, Dubinett SM and Keane MP: CXC chemokines: angiogenesis, immunoangiostasis, and metastases in lung cancer. Ann NY Acad Sci 1028: 351-360, 2004.

8. Singh S, Sadanandam A and Singh RK: Chemokines in tumor angiogenesis and metastasis. Cancer Metastasis Rev 26: 453-467, 2007.

9. Zlotnik A, Burkhardt AM and Homey B: Homeostatic chemokine receptors and organ-specific metastasis. Nat Rev Immunol 11: 597-606, 2011. 
10. Phillips RJ, Burdick MD, Lutz M, Belperio JA, Keane MP and Strieter RM: The stromal derived factor-1/CXCL12-CXC chemokine receptor 4 biological axis in non-small cell lung cancer metastases. Am J Respir Crit Care Med 167: 1676-1686, 2003.

11. Takanami I: Overexpression of CCR7 mRNA in nonsmall cell lung cancer: correlation with lymph node metastasis. Int J Cancer 105: 186-189, 2003.

12. Marchesi F, Piemonti L, Fedele G, et al: The chemokine receptor CX3CR1 is involved in the neural tropism and malignant behavior of pancreatic ductal adenocarcinoma. Cancer Res 68: 9060-9069, 2008.

13. Andre F, Cabioglu N, Assi H, et al: Expression of chemokine receptors predicts the site of metastatic relapse in patients with axillary node positive primary breast cancer. Ann Oncol 17: 945-951, 2006.

14. Marshall HM, Leong SC, Bowman RV, Yang IA and Fong KM: The science behind the 7th edition Tumour, Node, Metastasis staging system for lung cancer. Respirology 17: 247-260, 2012.

15. Dhillon T, Mauri FA, Bellezza G, et al: Overexpression of the mammalian target of rapamycin: a novel biomarker for poor survival in resected early stage non-small cell lung cancer. J Thorac Oncol 5: 314-319, 2010.

16. Shi SR, Key ME and Kalra KL: Antigen retrieval in formalinfixed, paraffin-embedded tissues: an enhancement method for immunohistochemical staining based on microwave oven heating of tissue sections. J Histochem Cytochem 39: 741-748, 1991.

17. Andreasson U, Ek S, Merz H, et al: B cell lymphomas express CX3CR1 a non-B cell lineage adhesion molecule. Cancer Lett 259: $138-145,2008$

18. White ES, Flaherty KR, Carskadon S, et al: Macrophage migration inhibitory factor and CXC chemokine expression in non-small cell lung cancer: role in angiogenesis and prognosis. Clin Cancer Res 9: 853-860, 2003.

19. Burger M, Glodek A, Hartmann T, et al: Functional expression of CXCR4 (CD184) on small-cell lung cancer cells mediates migration, integrin activation, and adhesion to stromal cells. Oncogene 22: 8093-8101, 2003.

20. Hartmann TN, Burger JA, Glodek A, Fujii N and Burger M CXCR4 chemokine receptor and integrin signaling co-operate in mediating adhesion and chemoresistance in small cell lung cancer (SCLC) cells. Oncogene 24: 4462-4471, 2005.

21. Raynaud CM, Mercier O, Dartevelle P, et al: Expression of chemokine receptor CCR6 as a molecular determinant of adrenal metastatic relapse in patients with primary lung cancer. Clin Lung Cancer 11: 187-191, 2010.

22. Shulby SA, Dolloff NG, Stearns ME, Meucci O and Fatatis A: CX3CR1-fractalkine expression regulates cellular mechanisms involved in adhesion, migration, and survival of human prostate cancer cells. Cancer Res 64: 4693-4698, 2004.

23. Kim M, Rooper L, Xie J, Kajdacsy-Balla AA and Barbolina MV: Fractalkine receptor CX3CR1 is expressed in epithelial ovarian carcinoma cells and required for motility and adhesion to peritoneal mesothelial cells. Mol Cancer Res 10: 11-24, 2012.

24. Matsubara T, Ono T, Yamanoi A, Tachibana M and Nagasue N: Fractalkine-CX3CR1 axis regulates tumor cell cycle and deteriorates prognosis after radical resection for hepatocellular carcinoma. J Surg Oncol 95: 241-249, 2007.
25. Jubb AM, Cesario A, Ferguson M, et al: Vascular phenotypes in primary non-small cell lung carcinomas and matched brain metastases. Br J Cancer 104: 1877-1881, 2011.

26. Baird AM, Gray SG and O'Byrne KJ: Epigenetics underpinning the regulation of the CXC $\left(\mathrm{ELR}^{+}\right)$chemokines in non-small cell lung cancer. PLoS One 6: e14593, 2011.

27. Mizuno T, Kawanokuchi J, Numata K and Suzumura A: Production and neuroprotective functions of fractalkine in the central nervous system. Brain Res 979: 65-70, 2003.

28. Hurst LA, Bunning RA, Couraud PO, et al: Expression of ADAM-17, TIMP-3 and fractalkine in the human adult brain endothelial cell line, hCMEC/D3, following pro-inflammatory cytokine treatment. J Neuroimmunol 210: 108-112, 2009.

29. Imaizumi T, Yoshida H and Satoh K: Regulation of CX3CL1/ fractalkine expression in endothelial cells. J Atheroscler Thromb 11: 15-21, 2004.

30. Nevo I, Sagi-Assif O, Meshel T, et al: The involvement of the fractalkine receptor in the transmigration of neuroblastoma cells through bone-marrow endothelial cells. Cancer Lett 273: 127-139, 2009.

31. Yamazaki K, Sugio K, Yamanaka T, et al: Prognostic factors in non-small cell lung cancer patients with postoperative recurrence following third-generation chemotherapy. Anticancer Res 30: 1311-1315, 2010.

32. Lee SJ, Namkoong S, Kim YM, et al: Fractalkine stimulates angiogenesis by activating the Raf-1/MEK/ERK- and PI3K/Akt/ eNOS-dependent signal pathways. Am J Physiol Heart Circ Physiol 291: H2836-H2846, 2006

33. Oh Y, Taylor S, Bekele BN, et al: Number of metastatic sites is a strong predictor of survival in patients with nonsmall cell lung cancer with or without brain metastases. Cancer 115: 2930-2938, 2009.

34. Mamon HJ, Yeap BY, Janne PA, et al: High risk of brain metastases in surgically staged IIIA non-small-cell lung cancer patients treated with surgery, chemotherapy, and radiation. J Clin Oncol 23: $1530-1537,2005$.

35. Chen AM, Jahan TM, Jablons DM, Garcia J and Larson DA: Risk of cerebral metastases and neurological death after pathological complete response to neoadjuvant therapy for locally advanced nonsmall-cell lung cancer: clinical implications for the subsequent management of the brain. Cancer 109: 1668$1675,2007$.

36. Simon GR and Turrisi A: Management of small cell lung cancer: ACCP evidence-based clinical practice guidelines (2nd edition). Chest 132: S324-S339, 2007.

37. Besse B, Massard C, Haddad V, et al: ERCC1 influence on the incidence of brain metastases in patients with non-squamous NSCLC treated with adjuvant cisplatin-based chemotherapy. Ann Oncol 22: 575-581, 2011

38. D'Haese JG, Demir IE, Friess H and Ceyhan GO: Fractalkine/ CX3CR1: why a single chemokine-receptor duo bears a major and unique therapeutic potential. Expert Opin Ther Targets 14: 207-219, 2010. 